American Journal of Environmental Sciences 4 (6): 721-726, 2008

ISSN 1553-345X

(C) 2008 Science Publications

\title{
Solute or Heat Transport in a Flat Duct
}

\author{
Elijah Johnson, Ayaovi D. Apeti, Sekeenia Haynes and Larry Robinson \\ Environmental Sciences Institute, Florida A and M University, Tallahassee, FL 32307-6600
}

\begin{abstract}
Steady state solute and heat transfer for laminar flow in a flat duct has been widely studied $^{[1-4]}$. The same problem in a circular tube is called the Graetz Problem ${ }^{[5,6]}$. The transfer rate of solute and heat from fluids is of importance in a number of processes, such as diffusion of drugs in the blood stream and the uptake of environmental contaminants by animals in aquatic media ${ }^{[7]}$. In this study the rate of solute or heat transfer from fluids was determined by solving the associated differential equation. Solution by the series approach in the complex plane was used with a series that had a gaussian factor. The eigenfunctions and eigenvalues involved were examined for two different sets of boundary conditions.
\end{abstract}

Key words: Boundary condition, eigenvalue, eigenfunction, flux, partial differential equation

\section{INTRODUCTION}

There are four types of boundary value problems that are of interest here. All of these boundary value problems have zero flux in the plane that is equidistant from and parallel to two boundary planes. The eigenfunctions and eigenvalues involved are examined here for two different sets of boundary conditions on this plane. One set of boundary conditions has been applied to the problem before ${ }^{[1-4]}$. The other has apparently not been. The eigenfunctions and eigenvalues are significantly different in each case. The condition at the other boundary is one of four types: (a) zero concentration, (b) zero flux, (c) constant flux, or (d) flux linearly proportional to the concentration at the boundary.

Treatments of these boundary value problems are given in the literature ${ }^{[1-4]}$. The treatments in much of the literature are based on only one of two possible choices for conditions that fulfill the zero flux condition on the central plane of the system. The condition used in the literature can fulfill the zero flux boundary condition only on the central plane. The second choice can fulfill the zero flux boundary condition anywhere in the system. Both conditions are examined here. For some choices of the two linearly independent solutions of the second order differential equation involved, only the second choice for the boundary condition is appropriate. The choice for the two linearly independent solutions determines the eigenvalues and eigenfunctions to be found.

The literature presents a discrete and apparently unbounded eigenvalue spectrum ${ }^{[1,2]}$. When the first choice for the boundary condition is applied to the two linearly independent solutions of the differential equation, the eigenvalue spectrum obtained is continuous and unbounded, because the first condition does not place restrictions on the coefficients of the linear combination of the two linearly independent solutions.

As the choice for the two linearly independent solutions of the differential equation changes, the eigenvalue spectrum changes. The most convenient solutions to choose are determined by the associated initial conditions. Convenience is often defined in terms of the rapidity of convergence of the representation of the solution to the associated initial value problem as a linear combination of eigenfunctions.

The system under examination here is a fluid mechanics system in which a fluid flowing with laminar motion between two parallel plates exchanges heat or mass with the plates. The rate of exchange can be determined by solving a partial differential equation. The system has been described ${ }^{[1-4]}$. The problem is to determine the concentration distribution and the transfer rate of mass or heat to the parallel plates. The system considered is similar to what is known as the Graetz Problem ${ }^{[8]}$.

This study proposes an alternative method of solving the partial differential equation involved. This method uses gaussian or trigonometric functions as factors in the series solution of the problem. Additionally, eigenvalues and eigenfunctions were determined. The study is important because it introduces a new pair of linearly independent solutions

Corresponding Author: Elijah Johnson, Environmental Sciences Institute, Florida A and M University, Tallahassee, FL 32307-6600 
to the differential equation and it uses a different zero flux condition in the central plane.

\section{MATERIALS AND METHODS}

The method used here factors out gaussian functions from the series solution of the problem. The removal of the gaussian factors from the series solutions leads to sine and cosine coefficients. The basic equation to be solved ${ }^{[1-4]}$ is:

$\mathrm{u}_{\mathrm{y}} \frac{\partial \mathrm{C}_{1}(\mathrm{x}, \mathrm{y})}{\partial \mathrm{y}}=\mathrm{D}_{1} \frac{\partial^{2} \mathrm{C}_{1}(\mathrm{x}, \mathrm{y})}{\partial \mathrm{x}^{2}}$

Where:

$$
\mathrm{u}_{\mathrm{y}}=-\frac{1}{2 \mu} \frac{\mathrm{dP}}{\mathrm{dy}}\left(\mathrm{h}^{2}-\mathrm{x}^{2}\right)
$$

Here :

$\mu \quad=$ The coefficient of viscosity of the fluid between the plates, $\mathrm{P}$ is the fluid pressure

$\mathrm{H}=$ Half the distance between the parallel plates, $D_{1}$ is the diffusion coefficient of the solute in the fluid

$\mathrm{x}$ and $\mathrm{y}=$ Denote Cartesian coordinates

The eigenfunction expansion solution of the problem is :

$\mathrm{C}(\mathrm{x}, \mathrm{y})=\sum_{\mathrm{m}=1}^{\infty} \mathrm{A}_{\mathrm{m}} \mathrm{G}\left(\mathrm{y}, \lambda_{\mathrm{m}}\right) \mathrm{F}\left(\mathrm{x}, \lambda_{\mathrm{m}}\right)$

where, the values of the expansion coefficients $A_{m}$ can be determined from a boundary condition on $y$. For example:

$\mathrm{C}(\mathrm{x}, 0)=\sum_{\mathrm{m}=1}^{\infty} \mathrm{A}_{\mathrm{m}} \mathrm{G}\left(0, \lambda_{\mathrm{m}}\right) \mathrm{F}\left(\mathrm{x}, \lambda_{\mathrm{m}}\right)$

The function $\mathrm{C}(\mathrm{x}, 0)$ is assumed to be known. The equations for $\mathrm{G}\left(\mathrm{y}, \lambda_{\mathrm{m}}\right)$ and $\mathrm{F}\left(\mathrm{x}, \lambda_{\mathrm{m}}\right)$ follow:

$\frac{\partial \mathrm{G}_{\mathrm{t}}(\mathrm{y}, \lambda)}{\partial \mathrm{y}}+\lambda^{2} \mathrm{G}_{1}(\mathrm{y}, \lambda)=0$

and

$\mathrm{D}_{1} \frac{\partial^{2} \mathrm{~F}_{1}(\mathrm{x}, \lambda)}{\partial \mathrm{x}^{2}}+\lambda^{2}\left(-\frac{1}{2 \mu} \frac{\mathrm{dP}}{\mathrm{dy}}\right)\left(\mathrm{h}^{2}-\mathrm{x}^{2}\right) \mathrm{F}_{1}(\mathrm{x}, \lambda)=0$

The solution to Eq. 5 is:
$\mathrm{G}_{\mathrm{l}}(\mathrm{y}, \lambda)=\mathrm{a}_{0} \exp \left(-\lambda^{2} \mathrm{y}\right)$

where, $\mathrm{a}_{0}$ is a constant of integration or boundary condition.

Remember that in some cases the conditions relevant to the associated physical problem is :

$\frac{\mathrm{dP}}{\mathrm{dy}} \leq 0$

The solution to Eq. 6 under the condition given by Eq. 8 will be given next. Let:

$\mathrm{F}_{1}^{\prime}(\mathrm{x}, \lambda)=\exp \left[-\frac{\lambda}{2}\left(\frac{-1}{2 \mu \mathrm{D}_{1}} \frac{\mathrm{dP}}{\mathrm{dy}}\right)^{1 / 2} \mathrm{x}^{2}\right] \mathrm{f}_{\lambda}(\mathrm{x})$

The differential equation obtained from Eq. 6 and 9 for $f_{\lambda}(x)$ is:

$\frac{\partial^{2} \mathrm{f}_{\lambda}(\mathrm{x})}{\partial \mathrm{x}^{2}}-2 \lambda \beta^{1 / 2} \mathrm{x} \frac{\partial \mathrm{f}_{\mathrm{\lambda}}(\mathrm{x})}{\partial \mathrm{x}}$

$+\left[\lambda^{2} \beta h^{2}-\lambda \beta^{1 / 2}\right] \mathrm{f}_{\lambda}(\mathrm{x})=0$

Where:

$\beta=\frac{-1}{2 \mu \mathrm{D}_{1}} \frac{\mathrm{dP}}{\mathrm{dy}}$

Let:

$f_{\lambda}(x)=\sum_{j=0}^{\infty} E_{j} x^{j}$

Then:

$\mathrm{E}_{\mathrm{j}+2}=\frac{(1+2 \mathrm{j}) \lambda \beta^{1 / 2}-\lambda^{2} \beta \mathrm{h}^{2}}{(\mathrm{j}+1)(\mathrm{j}+2)} \mathrm{E}_{\mathrm{j}}$

To examine several ways to express $F_{1}(x)$ in terms of a linear combination of two linearly independent functions, the solution is found for Eq. 6 for the case:

$\mathrm{F}_{1}^{\prime \prime}(\mathrm{x}, \lambda)=\exp \left[\frac{\lambda}{2}\left(\frac{-1}{2 \mu \mathrm{D}_{1}} \frac{\mathrm{dP}}{\mathrm{dy}}\right)^{1 / 2} \mathrm{x}^{2}\right] \mathrm{g}_{\lambda}(\mathrm{x})$

The differential equation for $\mathrm{g}_{\lambda}(\mathrm{x})$ is :

$\frac{\partial^{2} \mathrm{~g}_{\lambda}(\mathrm{x})}{\partial \mathrm{x}^{2}}+2 \lambda \beta^{1 / 2} \mathrm{x} \frac{\partial \mathrm{g}_{\lambda}(\mathrm{x})}{\partial \mathrm{x}}$

$+\left[\lambda^{2} \beta h^{2}+\lambda \beta^{1 / 2}\right] \mathrm{g}_{\lambda}(\mathrm{x})=0$ 
Let:

$$
g_{\lambda}(x)=\sum_{j=0}^{\infty} H_{j} x^{j}
$$

Then:

$$
\mathrm{H}_{\mathrm{j}+2}=\frac{-(1+2 \mathrm{j}) \lambda \beta^{1 / 2}-\lambda^{2} \beta h^{2}}{(\mathrm{j}+1)(\mathrm{j}+2)} \mathrm{H}_{\mathrm{j}}
$$

From Eq. 12, 13, 16 and 17, it follows that:

$g_{-\lambda}(x)=f_{\lambda}(x)$

when, $: \mathrm{E}_{\mathrm{j}}=\mathrm{H}_{\mathrm{j}}$.

So, from Eq. 8, 14 and 18:

$\mathrm{F}_{1}^{\prime \prime}(\mathrm{x} ;-\lambda)=\mathrm{F}_{1}^{\prime}(\mathrm{x}, \lambda)$

This indicates that $\mathrm{F}^{\prime}{ }_{1}(\mathrm{x}, \lambda)$ and $\mathrm{F}^{\prime}{ }_{2}(\mathrm{x},-\lambda)$ are not linearly independent. A complete solution of the differential equation has not been found until two linearly independent solutions have been chosen. that:

Because $\lambda$ occurs in Eq. 6 as $\lambda^{2}$, it is necessary

$\mathrm{F}_{1}^{\prime \prime}(\mathrm{x}, \lambda)=\mathrm{F}_{1}^{\prime \prime}(\mathrm{x},-\lambda)$

This and Eq. 19 imply that:

$\mathrm{F}_{1}^{\prime \prime}(\mathrm{x}, \lambda)=\mathrm{F}_{1}^{\prime}(\mathrm{x}, \lambda)$

Let:

$\mathrm{F}_{1}(\mathrm{x}, \lambda)=\mathrm{a}_{1} \exp \left(\frac{-\lambda}{2}\left(\frac{1}{2 \mu \mathrm{D}_{1}}\left|\frac{\mathrm{dP}}{\mathrm{dy}}\right|\right)^{1 / 2} \mathrm{x}^{2}\right) \mathrm{f}_{\lambda}(\mathrm{x})$

where, $\mathrm{a}_{1}$ is a constant. The boundary conditions are:

$\mathrm{F}_{1}(\mathrm{~h}, \lambda)=0$

and

$\left.\frac{\partial \mathrm{F}_{1}(\mathrm{x}, \lambda)}{\partial \mathrm{x}}\right|_{\mathrm{x}=0}=0$

The first boundary condition is met when:

$\mathrm{f}_{\lambda}\left(\mathrm{h}, \mathrm{E}_{0}\right)=0$

The second boundary condition is met when:

$$
\begin{aligned}
& -\left.\mathrm{a}_{1} \mathrm{x} \lambda \beta^{1 / 2} \exp \left(\frac{-\lambda}{2} \beta^{1 / 2} \mathrm{x}^{2}\right) \mathrm{f}_{\lambda}(\mathrm{x})\right|_{\mathrm{x}=0} \\
& +\left.\operatorname{ax}_{\mathrm{P}} \exp \left(\frac{-\lambda}{2} \beta^{1 / 2} \mathrm{x}^{2}\right) \frac{1 \mathrm{df} \mathrm{f}_{\lambda}(\mathrm{x})}{\mathrm{dx}}\right|_{\mathrm{x}=0}=0
\end{aligned}
$$

At $x=0$, this boundary is automatically satisfied when $f_{\lambda}(x)$ is an even function of $x$, so the stronger boundary condition implied by Eq. 26 has been ignored. To this point only the condition that $\mathrm{f}(\mathrm{x})$ is an even function of $\mathrm{x}$ has been used.

Equation 6 may be solved under the following condition":

$\frac{\mathrm{dP}}{\mathrm{dy}} \geq 0$

This condition is equivalency to that given by Eq. 18 when the coordinate system is rotated by $\pi$ about the xaxis. The solution to Eq. 6 under the condition given by Eq. 27 will be found next.

Let:

$\mathrm{F}_{1}^{\prime}(\mathrm{x}, \lambda)=\exp \left[-\frac{\lambda}{2}\left(\frac{-1}{2 \mu \mathrm{D}_{1}} \frac{\mathrm{dP}}{\mathrm{dy}}\right)^{1 / 2} \mathrm{x}^{2}\right] \mathrm{f}_{\lambda}(\mathrm{x})$

The differential equation for $\mathrm{f}_{\lambda}(\mathrm{x})$ of Eq. 28 is :

$\frac{\partial^{2} \mathrm{f}_{\lambda}(\mathrm{x})}{\partial \mathrm{x}^{2}}-2 \lambda \beta^{1 / 2} \mathrm{x} \frac{\partial \mathrm{f}_{\lambda}(\mathrm{x})}{\partial \mathrm{x}}+\left[\lambda^{2} \beta \mathrm{h}^{2}-\lambda \beta^{1 / 2}\right] \mathrm{f}_{\lambda}(\mathrm{x})=0$

Let:

$f_{\lambda}(x)=\sum_{j=0}^{\infty} E_{j} x^{j}$

The relationship for the expansion coefficients is :

$E_{j+2}=\frac{(1+2 j) \lambda \beta^{1 / 2}-\lambda^{2} \beta h^{2}}{(j+1)(j+2)} E_{j}$

Since Eq. 2) holds, it follows that:

$\left(\frac{-1}{2 \mu D_{1}} \frac{d P}{d y}\right)^{1 / 2}=\mathrm{i}\left(\frac{1}{2 \mu D_{1}}\left|\frac{d P}{d y}\right|\right)^{1 / 2}$

Where:

$\mathrm{i}=(-1)^{1 / 2}$

So: 
$\mathrm{F}_{1}^{\prime}(\mathrm{x}, \lambda)=\left[\begin{array}{c}\cos \left(\frac{\lambda}{2}\left(\frac{1}{2 \mu \mathrm{D}_{1}}\left|\frac{\mathrm{dP}}{\mathrm{dy}}\right|\right)^{1 / 2} \mathrm{x}^{2}\right) \\ -\mathrm{isin}\left(\frac{\lambda}{2}\left(\frac{1}{2 \mu \mathrm{D}_{1}}\left|\frac{\mathrm{dP}}{\mathrm{dy}}\right|\right)^{1 / 2} \mathrm{x}^{2}\right)\end{array}\right] \mathrm{f}_{\lambda}(\mathrm{x})$

Let:

$$
\mathrm{F}_{1}(\mathrm{x}, \lambda)=\frac{1}{2}\left[\mathrm{~F}_{1}^{\prime}(\mathrm{x}, \lambda)+\mathrm{F}_{1}^{\prime *}(\mathrm{x}, \lambda)\right]
$$

Here $\mathrm{F}^{\prime}{ }_{1}{ }^{*}$ is the complex conjugate of $\mathrm{F}^{\prime}{ }_{1}$. It follows that:

$$
\begin{aligned}
& \mathrm{F}_{1}(\mathrm{x}, \lambda)=\frac{1}{2}\left[\begin{array}{c}
\cos \left(\frac{\lambda}{2}\left(\frac{1}{2 \mu \mathrm{D}_{1}}\left|\frac{\mathrm{dP}}{\mathrm{dy}}\right|\right)^{1 / 2} \mathrm{x}^{2}\right) \\
-i \sin \left(\frac{\lambda}{2}\left(\frac{1}{2 \mu \mathrm{D}_{1}}\left|\frac{\mathrm{dP}}{\mathrm{dy}}\right|\right)^{1 / 2} \mathrm{x}^{2}\right)
\end{array}\right] \mathrm{f}_{\lambda}(\mathrm{x}) \\
&+\frac{1}{2}\left[\begin{array}{l}
\cos \left(\frac{\lambda}{2}\left(\frac{1}{2 \mu \mathrm{D}_{1}}\left|\frac{\mathrm{dP}}{\mathrm{dy}}\right|\right)^{1 / 2} \mathrm{x}^{2}\right) \\
+i \sin \left(\frac{\lambda}{2}\left(\frac{1}{2 \mu \mathrm{D}_{1}}\left|\frac{\mathrm{dP}}{\mathrm{dy}}\right|\right)^{1 / 2} \mathrm{x}^{2}\right)
\end{array}\right] \mathrm{f}_{\lambda}^{*}(\mathrm{x})
\end{aligned}
$$

Note that $E_{j}$ in Eq. 30 is given by:

$$
E_{j+2}=\frac{i \lambda|\beta|^{1 / 2}(1+2 j)+\lambda^{2}|\beta| h^{2}}{(j+1)(j+2)} E_{j}
$$

where, $|\beta|$ denotes the absolute value of the real number $\beta$. It follows from Eq. 35 that:

$$
\begin{aligned}
& \mathrm{F}_{1}(\mathrm{x}, \lambda)=\cos \left(\frac{\lambda}{2} \beta^{1 / 2} \mathrm{x}^{2}\right) \operatorname{Re}\left(\mathrm{f}_{\lambda}(\mathrm{x})\right) \\
& +\sin \left(\frac{\lambda}{2} \beta^{1 / 2} \mathrm{x}^{2}\right) \operatorname{Im}\left(\mathrm{f}_{\lambda}(\mathrm{x})\right)
\end{aligned}
$$

The boundary conditions are given by Eq. 23 and 24. The first boundary condition is met when:

$\tan \left(\frac{\lambda}{2}\left(\frac{1}{2 \mu D_{1}}\left|\frac{d P}{d y}\right|\right)^{1 / 2} h^{2}\right)=-\frac{\operatorname{Re}\left(f_{\lambda}(h)\right)}{\operatorname{Im}\left(f_{\lambda}(h)\right)}$

If the right-hand side of Eq. 39 is a constant, then the eigenvalues have a periodicity of $(4 \pi)$. In the case being treated here the right-hand side is a function of $\lambda$, so it is unlikely that the eigenvalues are periodic.

The second boundary condition is met when:

$$
\begin{aligned}
\left.x \sin \left(\frac{\lambda}{2} \beta^{1 / 2} x^{2}\right)\left[\begin{array}{l}
-\lambda \beta^{1 / 2} \operatorname{Re}\left(\mathrm{f}_{\lambda}(\mathrm{x})\right) \\
\left.+\operatorname{Im}\left(\frac{\left.1 \mathrm{~d} \mathrm{f}_{\lambda} \mathrm{x}\right)}{\mathrm{dx}}\right)\right)
\end{array}\right]\right|_{\mathrm{x}=0} \\
+\left.\operatorname{xcos}\left(\frac{\lambda}{2} \beta^{1 / 2} \mathrm{x}^{2}\right)\left[\begin{array}{l}
\lambda \beta^{1 / 2} \operatorname{Im}\left(\mathrm{f}_{\lambda}(\mathrm{x})\right) \\
+\operatorname{Re}\left(\frac{1}{\mathrm{x}} \frac{\mathrm{df}(\mathrm{x})}{\mathrm{dx}}\right)
\end{array}\right)\right|_{\mathrm{x}=0}=0
\end{aligned}
$$

Equation 40 is equivalent to:

$\lambda \beta^{1 / 2} R_{0} \sin \left(\theta_{0}\right)+2 R_{2} \cos \left(\theta_{2}\right)=0$

or

$\theta_{0}=\arctan \left(-\frac{\sigma^{2}}{2}\right)$

Where:

$\sigma^{2} \equiv \frac{\lambda}{2}\left(\frac{1}{2 \mu D_{1}}\left|\frac{d P}{d y}\right|\right)^{1 / 2} h^{2}=\lambda \beta^{1 / 2} h^{2}$

The quantities $R_{0}$ and $R_{2}$ are the real parts of $E_{0}$ and $E_{2}$, respectively. The quantities $E_{0}$ and $E_{2}$ are coefficients in the series expansion given by Eq. 30 .

The function $f_{\lambda}(x)$ may be found using the following prescription:

$$
\begin{aligned}
& \mathrm{f}_{\lambda}(\mathrm{x})=\sum_{\mathrm{j}=0}^{\infty} \mathrm{E}_{\mathrm{j}} \mathrm{x}^{\mathrm{j}}=\sum_{\mathrm{j}=0}^{\infty}[\operatorname{Re}(\underset{\mathrm{f}}{\mathrm{f}}) \\
& \left.\left.+\mathrm{i} \operatorname{Im}\left(\mathrm{E}_{\mathrm{j}}\right)\right] \mathrm{x}^{\mathrm{j}}=\operatorname{Re}\left(\mathrm{f}_{\lambda}(\mathrm{x})\right)+\mathrm{i} \operatorname{Imf}_{\lambda}(\mathrm{x})\right)
\end{aligned}
$$

Where:

$$
\operatorname{Re}\left(f_{\lambda}(x)\right)=\sum_{j=0}^{\infty} \operatorname{Re}\left(E_{j}\right) x^{j}
$$

and

$$
\operatorname{Im}\left(f_{\lambda}(x)\right)=\sum_{j=0}^{\infty} \operatorname{Im}\left(E_{j}\right) x^{j}
$$

The complex coefficients $\mathrm{E}_{\mathrm{j}}$ may be expressed as :

$$
\begin{aligned}
& E_{j+2}=\frac{i \lambda|\beta|^{1 / 2}(1+2 j)+\lambda^{2}|\beta| h^{2}}{(j+1)(j+2)} E_{j} \\
& =r_{j+2} \exp \left(i \phi_{j+2}\right) R_{j} \exp \left(i \theta_{j}\right)
\end{aligned}
$$


Am. J. Environ. Sci., 4 (6): 721-726, 2008

Where:

$$
\begin{aligned}
& R_{j+2}=r_{j+2} R_{j} \\
& \theta_{j+2}=\phi_{j+2}+\theta_{j} \\
& \operatorname{Re}\left(E_{j+2}\right)=R_{j} \cos \left(\theta_{j+2}\right)
\end{aligned}
$$

and

$\operatorname{Im}\left(E_{j+2}\right)=R_{j} \sin \left(\theta_{j+2}\right)$

Here :

$\mathrm{R}_{0}=\mathrm{E}_{0}$

and

$\mathrm{R}_{1}=\mathrm{E}_{1}$

The value of $\theta_{0}$ must be specified. The values of $\mathrm{r}_{\mathrm{j}+2}$ and $\phi_{\mathrm{j}+2}$ are given by:

$r_{j+2}=\frac{1}{(j+1)(j+2)}\left[\lambda^{2}|\beta|(1+2 j)^{2}+\lambda^{4}|\beta|^{2} h^{4}\right]^{1 / 2}$

and

$$
-\phi_{j+2}=\operatorname{arcos}\left(\frac{(\lambda h)^{2}|\beta|}{(j+1)(j+2) r_{j+2}}\right)
$$

The standard procedure for finding eigenvalues is to use one of the boundary conditions to place limitations on the values of the undetermined parameters. The undetermined parameters here are $\mathrm{R}_{0}$ and $\theta_{0}$. The next step is to place limitations on the eigenvalues using the remaining boundary condition. This procedure was used to find the condition on $\lambda$ given by Eq. 42. The value of $R_{\theta}$ can be found by imposing a normalization condition. The allowed values of $\theta_{0}$ are determined from the second boundary condition. The second boundary condition imposes the condition on $\theta_{0}$ given by Eq. 42. Values for $\lambda$ are then found for a given value of $\theta_{0}$. The dependence of $\lambda$ on $\mathrm{R}_{0}$ and $\theta_{0}$ are given by Eq. 37, 39 and 42 .

\section{RESULTS}

The results in the Table 1 were obtained using the following condition:

$\mathrm{E}_{1}=0$
Table 1: Eigenvalues for three different choices for the two linearly independent solutions to Eq. 6. Column I: Gaussian factor, Eq. 9. Column II: Trigonometric functions factors, Eq. 38, with $\theta_{0}=0$. Column III: Trigonometric function factors, Eq. 38 , with $\tan \left(\theta_{0}\right)=-\sigma^{2} / 2$

\begin{tabular}{llll}
\hline \multirow{2}{*}{ Eigenvalue } & \multicolumn{2}{l}{ Combination of linearly independent functions } \\
No. & I & II & III \\
\hline 1 & 1.682 & 1.571 & 1.059 \\
2 & 5.670 & 4.712 & 6.333 \\
3 & 9.668 & 7.854 & 12.579 \\
4 & 13.668 & 10.996 & 18.855 \\
5 & 17.667 & 14.137 & 25.136 \\
6 & 21.667 & 17.279 & 31.418 \\
7 & 25.667 & 20.420 & 37.700 \\
8 & 29.667 & 23.562 & 43.982 \\
9 & 33.667 & 26.704 & 50.266 \\
10 & 37.667 & 29.845 & 56.549 \\
\hline
\end{tabular}

This choice for $E_{1}$ means that $f(x)$ is an even function of $\mathrm{x}$. The eigenvalues and the eigenfunctions were found numerically. The results for eigenvalues are given in the table. The eigenvalues for three cases are given. The results in Column I of the table are for the case that has been discussed in the literature ${ }^{[1-4]}$. The results in Column I are for a "weak" zero flux condition in the central plane with the solution of Eq. 6 given by Eq. 9, 12, 13, 23 and 24. The values in Column I are the same as those in the literature ${ }^{[1,2]}$. The results in Column II are also for a "weak" zero flux condition in the central plane with the solution of Eq. 6 given by Eq. 38,39 and $45-55$ with $\theta_{0}=0$. The results in Column III are also for a "strong" zero flux condition in the central plane with the solution of Eq. 6 given by Eq. 38, 39, 42 and 45-55.

\section{CONCLUSION}

The solution to one of four boundary value problems of interest is presented here. The method used to solve the boundary value problem treated here is applicable to the other three boundary value problems.

The approach to solving the problem differs in several ways from the way that the problem has been treated in the literature. First, a new form for the solution is introduced. Second, a different zero flux on the central plane boundary condition is used. Before this study only one set of eigenfunctions had been presented. The most convenient set of eigenvalues and eigenfunction to use depends on the specific initial value problem being treated.

\section{ACKNOWLEDGEMENT}

The study reported here was supported by the National Oceanic and Atmospheric Administration 
Environmental Cooperative Science Center under grant number NA-17-AE1642.

\section{REFERENCES}

1. Sellars, J.R., M. Tribus and J.S. Klein, 1956. Heat transfer to laminar flow in a round tube or flat conduit-The Graetz problem extended. Am. Soc. Mech. Eng. Transfer, 78: 441-448.

2. Brown, G.M, 1960. Heat or mass transfer in a fluid in laminar flow in a circular or flat conduit. Am. Inst. Chem. Eng. J., 6: 179-183.

3. Colton, C.K., K.A. Smith, P. Stroeve and E.W. Merrill, 1971. Laminar flow mass transfer in a flat duct with permeable walls. Am. Inst. Chem. Eng. J., 17: 773-780.

4. Walker, G. and T. Davies, 1974. Mass transfer in laminar flow between parallel permeable plates. Am. Inst. Chem. Eng. J., 20: 881-889.
5. Barron, R.F., X. Wang, R.O. Warrington and T. Ameel, 1996. Evaluation of the eigenvalues for the graetz problem in slip-flow. Intl. Commun. Heat Mass Transfer, 23: 563-574.

6. Mikhailov, M.D. and R.M. Cotta, 1997. Eigenvalues for the Graetz Problem in slip-flow. Intl. Commun. Heat Mass Transfer, 24: 449-451.

7. Barber, M.C., L.A. Saurez and R.R. Lassiter, 1991. Modeling bioaccumulation of organic pollutants in fish with an application to PCBs in Lake Ontario Salmonids. Can. J. Fish. Aquat. Sci., 48: 318-337.

8. Graetz, L., 1985. Uber die Warmelietungsfahigkeit von Flussigkeiten. Ann. Phys. Chem., 25: 337-357. 